\title{
Ferdinand Gonseth (1890-1975): Mathematik und Wirklichkeit
}

\author{
Ein Stück Geschichte der Wissenschaftsphilosophie in der Schweiz
}

\section{ANDRE MERCIER}

Es soll hier weder das Denken Gonseth's bis ins einzelne analysiert, noch sein Werk ganz allgemein beschrieben werden. Mein Berner Kollege Henri Lauener hat dies bereits weitgehend getan in einem $1971^{1}$ erschienenen Artikel, welcher einen Gesamtüberblick über die Arbeiten im Gebiete der Wissenschaftstheorie in der Schweiz vermitteln soll. Der Überblick ist allerdings insofern unvollständig, als er die ganze Entwicklung in der eigentlichen Westschweiz unberücksichtigt läßt und außerdem $z \mathfrak{u}$ einem recht späten Zeitpunkt einsetzt. Im übrigen enthält der genannte Artikel die vollständige Publikationsliste F. Gonseth's (und auch weiterer Autoren).

Meine Absicht besteht nun darin, hier den Eindruck wiederzugeben, den Ferdinand Gonseth als Mensch mir vermittelt hat, und zu versuchen, ihm seinen Platz in der gesamten Philosophie in der Schweiz und in der Welt zuzuweisen.

In seinem Schauspiel Wilhelm Tell läßt Schiller die alten Schweizer als ein ,einig Volk" auftreten ${ }^{2}$. Der Held des Dramas ist zwar ein ausgesprochener Individualist, jedoch bereit, sein Leben für die andern hinzugeben. Das Motto „einer für alle, alle für einen" gilt heute noch in der kleinen Schweiz. Das Land ist arm von Natur aus, es wächst kaum mehr als Gras, und von Bodenschätzen findet sich keine Spur; der Winter ist hart, sogar in der Ebene, die sich zwischen den Alpen und dem Jura ausdehnt. Jura ist ein Wort keltischen Ursprungs und bezeichnet von Wäldern bedeckte Berge; man findet das Wort als Name einer Insel nordwestlich von Schottland. Diese Insel ist ebenfalls von Bergwäldern bedeckt.

1 Vgl. diese Zeitschrift Band II, 1971, S. 291-317 Bericht: über die,,Wissenschaftstheorie in der Schweiz", sowie daselbst, S. 340-351: Veröffentlichungen schweizerischer Wissenschaftstheoretiker $I$.

2 Friedrich Schiller, Withelm Tell, 2. Aufzug. 2. Szene. Alle (sich die Hand reichend): „Wir sind $e$ in Volk, und einig wollen wir handeln." (Schillers sämtliche Werke in 12 Bänden, J. G. Cottascher Verlag, 6. Band, Stuttgart und Tubingen 1847, S. 64). 
Die Schweizer, von denen Schiller spricht, sind wohl einig - trotz der Sprach- und Konfessionsunterschiede, welche sich im Laufe der Geschichte ergaben -, sie sind gleichzeitig auch große Individualisten, doch - im Gegensatz zu den lateinischen Ländern - sind sie schweigsam, haben wenig übrig für Rhetorik und neigen zum Puritanismus - nicht in der angelsächsischen Deutung, welche dieses Wort im Jahrhundert der Reform in England oder unter der Herrschaft der Königin Viktoria erhalten hat, sondern im ursprünglichen Sinne der Reinheit der Beziehungen zwischen dem, was man denkt und dem, was man tut.

Außerdem ist der Schweizer von Grund aus großzügig: großzügig nicht so sehr, wenn es um sein Geld geht (denn da ist er, im Gegenteil, sehr bedächtig), vielmehr aber in seiner persönlichen Haltung gegenüber andern Personen. Dies ist einer der Gründe, weshalb er oft ein guter Pädagoge ist.

Andererseits ist der Schweizer weder sehr der Mystik zugetan, noch ein großer Poet, noch ein glänzender Logiker, drei Eigenschaften, welche eher den Franzosen von Pascal bis Valéry zukommen.

Das bisher Gesagte läßt sich leicht in die Feststellung überführen, daß die Schweizer ein Volk von Technikern bilden. Der Schweizer ist Ingenieur, weil er ein sehr begabter Mechaniker ist, der Franzose ist Ingenieur dank seiner mathematischen Begabung, der Deutsche aufgrund seiner Ausdauer und der Engländer, weil er seine Zeit damit verbringt, seine Maschinen zu vervollkommnen.

Der Schweizer steht mit beiden Füßen auf dem Erdboden.

*

Was hat dies alles mit Ferdinand Gonseth und der Philosophie der Wissenschaften $z u$ tun?

Vorerst einmal soviel, daß Gonseth aus dem Jura stammte, und mit ihm zwei andere seiner Generation, welche zu ihren Lebzeiten ihn an Brillanz übertrafen, nämlich Rolin Wavre und Gustave Juvet. Die beiden letzteren hatten sich in der französischen Schule der Mathematik ausgebildet, während die Ausbildung Gonseth's eher germanisch ausgerichtet war.

Vor der Zeit der Gruppe von Schweizer Mathematikern und Gelehrten, zu welcher diese drei Männer und auch andere noch gehörten, die sich den epistemologischen Problemen zugewandt haben (wir möchten Einstein und Polya dazuzählen, welche als junge Männer in die Schweiz gekommen sind und hier studiert haben, während Hermann Weyl, W. Pauli oder Paul Bernays mit ganz andern Voraussetzungen aus dem Ausland gekommen sind), - vor dieser Gruppe also wurde in der Schweiz die Mathematik vor allem als ein Unterrichtsfach angesehen, welches den Ingenieuren oder den zukünftigen Gymnasiallehrern dienlich war. Die großen Schweizer Mathematiker wie Euler, Bernoulli, Sturm ... gehören früheren Perioden an, und einzig Schlaefli war ein typischer 
autochthoner Schweizer geblieben. General Dufour, Vorkämpfer der darstellenden Geometrie in der Tradition von Monge, war Polytechniker und mit Kaiser Napoleon III befreundet.

Vor Beginn des 20. Jahrhunderts hat es keine gesamtschweizerische Tradition gegeben, von welcher aus sich philosophische Gedanken über Mathematik oder über positive Wissenschaften hätten entwickeln können. Außerdem haben die Schweizer Philosophen - und sie waren damals nicht zahlreich - sich hauptsächlich aus der Westschweiz rekrutiert; sie waren und sind noch heute mehrheitlich „Philosophen von irgendetwas", viel eher als reine Metaphysiker. Sicher, es hat auch Metaphysiker gegeben, Frederic Amiel im 19. Jahrhundert zum Beispiel, oder besser noch Charles Werner, in der ersten Hälfte des 20. Jahrhunderts, in Genf. Doch Amiel hatte in Deutschland studiert, und die Familie Werner stammte von der baltischen Küste. Werner's Zeitgenosse Arnold Reymond aus Lausanne hingegen gehörte zu jenen typisch schweizerischen Denkern, welche Religionsphilosophen, Sprachphilosophen oder Wissenschaftsphilosophen sind, oder gar Mediziner mehr oder weniger psychologischer Prägung ${ }^{3}$. So war die Sprachphilosophie im Sinne von Linguistik vertreten durch Leute wie Ferdinand de Saussure, Charles Bailly, Anton Marty, die Religionsphilosophie durch eine ganze Theologenschule, von welcher sich Männer wie Arnold Reymond loslösten, weil sie sich durch das Mysterium a-religiöser Art angezogen fühlten, das der sensationelle epistemische Erfolg der positiven Wissenschaft darbot, und so kam es auch, daß die aufkommende wissenschaftliche Psychologie des ausgehenden 19. Jahrhunderts sich in Genf in einer Schule konstituierte. Und man versteht, daß die eigentliche Philosophie der Wissenschaften zu Beginn dieses Jahrhunderts entstanden ist, und zwar ganz besonders in den Kreisen von Gelehrten, die sich mit exakten Wissenschaften befaßten, in der Westschweiz.

Nur im Zusammenhang mit der Psychologie hat diese Entwicklung alsbald ein Gegenstück in der deutschen Schweiz gefunden, mit der Schule von Carl Jung in Zürich.

Doch ist es in erster Linie in Verbindung mit den exakten Wissenschaften, daß sich in der Westschweiz die ersten Versuche einer Wissenschaftstheorie feststellen lassen. "Wissenschaftstheorie" ist die Bezeichnung, die man heutzutage verwendet; damals sprach man, ohne deutlich zu unterscheiden, von Philosophie der Wissenschaften und sogar von Erkenntnistheorie, denn für die Denker, auf welche wir hier anspielen, war ganz automatisch die Erkenntnis identisch mit wissenschaftlicher

${ }^{3}$ Henri Reverdin, der „Philosoph des Roten Kreuzes“, war Moralist. Henri Miéville, und vor allem Paul Häberlin waren schon mehr auf die Metaphysik ausgerichtet, obgleich der letztere eher ein Pädagoge war. Später war der ũberzeugte Platoniker Hermann Gauss ein Metaphysiker, und die Existenzphilosophen, Carlo Sganzini nach seiner Weise und Heinrich Barth ganz besonders, hatten vielleicht eine metaphysische Ader, wie auch Georges Mottier, obwohl Ästhetiker. Hans Barth seinerseits war ein Philosoph der Politik. Ich zitiere hier keine lebenden Schweizer Philosophen. 
Erkenntnis, und für sie erhielt auch das Wort Wissenschaft den französischen oder englischen Sinn von Naturwissenschaft - wobei die Mathematik eine ,zu überprüfende" Rolle spielte - und nicht die deutsche Sinngebung, welche vor allem seit Dilthey alles und zugleich nichts besagen will, denn im Deutschen ist alles Wissenschaft, einschlieBlich der Geisteswissenschaften.

Selbstverständlich kann man den Einfluß eines Pierre Duhem (den die amerikanischen Epistemologen kürzlich wiederentdeckt haben) oder eines Henri Poincaré auf diese Entwicklung nicht leugnen, noch darf man hier einen Ernst Mach übergehen, dessen Buch Die Mechanik in ihrer Entreickelung historisch-kritisch dargestellt (Leipzig 1883) 1904 in Paris in französischer Übersetzung erschien (bei Hermann: Buchhandlung S.M. des Königs von Schweden und von Norwegen). $\mathrm{Zu}$ jener Zeit unterrichtete Charles Cailler in Genf theoretische Mechanik, und jene, die ihn gehört haben - so z.B. mein Vater - hielten ihn für einen außergewöhnlichen Pädagogen und eine faszinierende Persönlichkeit. Es war auch die Zeit, da man sich $z u$ fragen begann ob die positivistische Klassifikation der Wissenschaften, welche in aufsteigender Folge der Zahl, dem Raum, der Zeit, der Masse, der Materie im allgemeinen, dem Leben, der Seele und der Gesellschaft (als ob dies vergleichbare „Kategorien“ wären) die Arithmetik, die Geometrie, die Kinematik, die Mechanik, die Physik, die Biologie, die Psychologie und die Soziologie zuordnete (als ob es sich hier um vergleichbare "Wissenschaften" handelte) - ob diese Klassifikation haltbar sei. Die Veröffentlichung der speziellen Relativitätstheorie durch Einstein im Jahre 1905, mehr noch als jene der Quantentheorie des schwarzen Körpers durch Planck 1900, mußte dieser Klassifikation den ersten Dolchstich versetzen. Und im Jahre 1910 gelang Charles Eugene Guye und seinen Mitarbeitern (insbesondere Lavanchy) mittels Beta-Strahlen die erste experimentelle, bis ins einzelne gehende Verifikation der speziellen Relativitätstheorie wie sie sich in der Abhängigkeit zwischen Masse und Geschwindigkeit manifestiert. Ch. E. Guye war übrigens der erste Schweizer, welcher eine "Philosophie der Physik" ins Leben rief, und zwar durch seine Arbeiten über Sinn und Bedeutung der sogenannten Physiko-Chemie in der Erklärung des Phänomens Leben, und durch seine Gedanken über die pythagoräische Natur der wissenschaftlichen Forschung und über die Verbindungen zwischen Wissenschaft und Kunst.

In diesem Klima des beginnenden Jahrhunderts hat in der Schweiz die Diskussion über die Wissenschaft eingesetzt und haben spezifische Forschungen in diesem Gebiet ihren Anfang genommen. Zum selben Zeitpunkt übrigens haben die Philosophen in der Westschweiz, gleich wie die französischen und angelsächsischen Philosophen, ob sie nun Berufsphilosophen, Amateurphilosophen oder Spezialisten in einer bestimmten Wissenschaft waren, ein unbändiges Bedürfnis verspürt, sich zusammenzufinden, um gemeinsam zu diskutieren, und sie haben die philosophische Gesellschaft der Westschweiz gegründet. Die Zeit von 
Frederic Amiel, dem unsteten und einsamen verspäteten Romantiker, welchem meine Großtante als eine Art Privatsekretärin in tiefer Freundschaft verbunden war, diese Zeit war vorüber. In der deutschen Schweiz hat sich ein derartiges Bedürfnis nie geltend gemacht, vielleicht deshalb, weil die Philosophie an den Universitäten dort immer eher deutschen Professoren als Schweizern anvertraut worden ist.

Zusammenfassend steht also fest, daß sich in der deutschen Schweiz nichts entwickelt hat, das dazu angetan gewesen wäre, den Boden für die Entfaltung einer Philosophie der Wissenschaften vorzubereiten.

Als mein Vater aus Zürich zur ückkehrte, wo er einige Jahre vor Einstein seine Studien an der Eidgenössischen Technischen Hochschule absolviert hatte, in Gesellschaft z.B. von Pierre Ceresole, dem späteren großen antimilitaristischen Apostel des Zivildienstes und Freund Gandhis, da stellte er diese Differenz in der Geisteshaltung fest. Ceresole, der seinerzeit den Lehrstuhl für Mechanik an der E.T.H. abgelehnt hat, um sich vollständig seinem humanitären Werk zu widmen, verspürte den Unterschied noch stärker, denn er hatte den Kopf eines Wissenschaftstheoretikers (er hat unzählige fast unleserliche Notizen hinterlassen über die Grundlagen der Wahrscheinlichkeitsrechnung).

Seit den Zwanzigerjahren haben sich dann in der Folge die philosophischen Diskussionen ausgeweitet, an denen die Vertreter der physikalisch-mathematischen Wissenschaften und der Psychologie ausnehmend starken Anteil nahmen. Rolin Wavre hatte den Lehrstuhl für Mechanik in Genf übernommen und Gustave Juvet jenen für Analysis in Neuenburg, und dann denjenigen für mathematische Physik in Lausanne. Wavre hatte in Paris, wo er unter dem Einfluß von Jean Cavaillès (einem der subtilsten Vertreter der mathematischen Philosophie in Frankreich und außerdem zum Mystizismus neigend) gestanden hatte, sich glänzend hervorgetan. Er wandte sich dem damals verbreiteten Nachdenken über die sogenannten Grundlagen der Mathematik zu und erforschte die Frage, inwiefern die Mathematik ,erfindet" oder ,entdeckt". Juvet andererseits fand alsbald Anerkennung als Bahnbrecher für ein Philosophieren in französischer Sprache über die neuen physikalischen Theorien, insbesondere über die Quantenphysik. (Meyerson, ur prünglich Chemiker, ist nicht über eine klassische Wissenschaft hinausgekommen, Bachelard besa $B$ keine wissenschaftliche Ausbildung und Louis de Broglie ist erst später gekommen). Hinzuzufügen ist Jean Piaget, der als Zoologe begann, sich mit Psychologie auseinanderzusetzen und schließlich zum großen Förderer der genetischen Epistemologie wurde. Alle diese Gelehrten, einschließlich Samuel Gagnebin in Neuenburg und Perceval Frutiger in Genf, fanden sich zusammen, und Arnold Reymond diente ihnen, mehr als sonst irgendjemand, als philosophischer Mentor.

Ferdinand Gonseth, der - obwohl Westschweizer - seine akademische Ausbildung in der deutschen Schweiz absolviert hatte und alsbald auf den Lehrstuhl für Geometrie nach Bern berufen wurde, war zu neugierig und $\mathrm{zu}$ intelligent, um nicht zu bemerken, daß ,,jenseits der Saane", wie 
man sagt, d.h. westlich der Sprachgrenze (Gonseth war französischer Muttersprache, jedoch durch seinen Bildungsgang gleichsam zweisprachig) kennenswerte Dinge vor sich gingen. Er ist von jener Begeisterung erfaßt worden, welche die französischsprechenden Mathematiker und Theoretiker beseelte und dazu antrieb, über den Rahmen der reinen wissenschaftlich-technischen Forschung hinauszugehen. Es versteht sich, $\mathrm{da} B$ Gonseth hierzu bereits eine natürliche Neigung besaß. Außerdem zwang ihn seine beinah völlige Blindheit, welche er sich in seiner Jugend durch eine Vergiftung zugezogen hatte, dazu, mehr mit dem Geist als mit den Augen zu arbeiten und eher über das Wesen dessen, was er lehrte, nachzudenken als sich technischen Forschungsarbeiten zu widmen.

Doch in der deutschen Schweiz fand er nicht die seinen Wünschen und Erwartungen entsprechende Atmosphäre. Er mußte sie also schaffen, woraus sich, ohne Übertreibung gesagt, eine dialektische Situation ergeben hat.

Als großer Individualist und ziemlicher Puritaner im oben beschriebenen Sinne - er entstammte übrigens, wie praktisch alle hier erwähnten Denker, dem Protestantismus, aber zu einer Zeit, da man nicht mehr an das biblische Wort ,glauben" konnte wie noch damals, als meine Großtante in ihrer Schule die heilige Schrift als Grundlage der Kultur eines calvinistischen Landes hochhielt und junge Mädchen aus ganz Europa in diesem Sinne unterrichtete - hat sich Gonseth wahrscheinlich veranlaßt gefühlt, eine eigene, ganz persönliche Basis zu erstellen für das Nachdenken über das Wesen der Mathematik und insbesondere der Geometrie.

Ich glaube nicht, da $\beta$ jemals ein Wissenschaftstheoretiker rein helvetischer Zugehörigkeit Platoniker ${ }^{4}$ (Identifikation der Idee mit dem, was wirklich ist), oder Aristoteliker gewesen ist ${ }^{5}$ (Identifikation dessen, was ist, mittels Analyse dessen, was zu sein scheint) - man denke an Raphaels Schule von Athen, wo die rechte Hand des Einen auf den Himmel und diejenige des Andern auf die Erde zeigt -, oder gar Kantianer, denn keiner von uns hat je versucht, etwas apodiktisch festzulegen. Für Juvet waren Mathematik und Poesie die beiden notwendigen und komplementären Pole der sprachlichen Äußerung. Für Wavre bedeutete die Mathematik den Ort der Begegnung zwischen menschlichem Erfindungsreichtum und Entdeckergeist. Für Piaget ist die Aneignung von Kenntnissen und von Erkenntnismitteln genetisch.

Für Gonseth drängt sich die Mathematik dem Geist auf; sie taucht aus der Wiklichkeit auf und formt mit ihr eine Dialektik, die Dialektik des Konkreten und Abstrakten, des Natürlichen und Axiomatischen, der Materie und des Denkens. Das Denken versucht immer exakter zu werden,

4 Siehe die Ausnahmen in Anm. 3.

5 Abgesehen von den katholischen Philosophen neo-thomistischer Prägung (ausgenommen vielleicht die Benediktiner und die Kanoniker von St. Maurice), sowie von gewissen Phänomenologen der von Pierre Thévenaz gegründeten Richtung. 
aber die Wirklichkeit kümmert das nicht, denn sie ist ganz einfach die Wirklichkeit. Die Wirklichkeit verpflichtet also, in dieser Dialektik, das Denken, sich wiederzudenken, sich zu überprüfen, sich zu korrigieren und sich anzupassen: offen zu bleiben. Ein Denken, das sich schließt, ist dazu verurteilt zu erstarren. Daher die gonsethsche ,offene Methodologie“, welche eine Haltung fordert, die es jederzeit erlaubt, sein Denken aufgrund von neu Hinzugelerntem zu revidieren. Weil es sich um eine "Methode" handelt, nennt Gonseth seine Wissenschaftstheorie oder Wissenschaftsphilosophie eine "Methodologie“. Und dieses ",neu Hinzugelernte" stammt übrigens aus den Wissenschaften, weil sie es sind, die die Wirklichkeit untersuchen, so da $\beta$ die Wissenschaft der Philosophie als Kriterium dient und nicht umgekehrt. Gonseth hätte den ,,philosophischen Imperativ“", von welchem Alwin Diemer gegen $1975 \mathrm{zu}$ sprechen beliebte, nicht anerkannt. In den Fünfzigerjahren bemerkte Hermann Gauss, Inhaber des Lehrstuhles für Philosophie in Bern: „Ferdinand Gonseth schließt sich mehr und mehr in das System seiner offenen Philosophie ein". Eine spitze Bemerkung, doch nicht ohne ein Körnchen Wahrheit, denn es gibt in dieser Forderung, daB die Philosophie sich als etwas Offenes zu betrachten habe, irgendetwas Abschließendes. Aber dies ist der Dialektik, einer Dialektik der Praxis und der Theorie, vielleicht nicht fremd.

Nach ungefähr zehnjähriger Tätigkeit in Bern, wo er wahrscheinlich bei seinen Schülern - im Gebiete des Nachdenkens über die Mathematik mehr Anklang fand (besonders beim Physiker Hans König, der später Direktor des Eidgenössischen Amtes für $\mathrm{MaB}$ und Gewicht werden sollte, sowie beim Statistiker Arthur Linder, der später auf den Lehrstuhl für mathematische Statistik nach Genf berufen wurde) als bei seinesgleichen, übernahm Gonseth den Lehrstuhl für Mathematik für Französischsprechende an der Eidgenössischen Technischen Hochschule in Zürich: ein geistiger Klimawechsel, größere Entfernung von der Westschweiz, und Annäherung an Deutschland in intellektuellen Belangen. Wenn die Universität Bern ihrerseits stets darauf geachtet hat, einige Gelehrte zu verpflichten, welche dem lateinischen Kulturkreis zugehören, so war dies, aufgrund eines Bundesgesetzes, bei der E.T.H. noch ausgeprägter der Fall, allerdings nicht im Hinblick auf die Pflege philosophischen Denkens. Erst später haben sich Hermann Weyl und Paul Bernays in Zürich niedergelassen, und noch später erst hat Wolfgang Pauli sein Interesse für die Psychoanalyse und deren anscheinende Komplementarität mit der Physik bekundet. Deshalb auch haben alle Kontakte, die Gonseth in Zürich mit seinen Kollegen auf epistemologischem Gebiet hat pflegen können, durch das Medium der deutschen Sprache und einer gegenseitigen Anpassung an die deutsche Denkweise hindurchgehen müssen ${ }^{6}$.

- Der Nachfolger von Gonseth auf dem Lehrstuhl für Geometrie in Bern, Willy Scherrer, war offensichtich sehr an epistemologischen Problemen interessiert, insbesondere im Zusammenhang mit der Geometrie und mit bestimmten Aspekten der theoretischen Physik. 
Was mir nun hier zu tun bleibt ist also, auf die Stellung aufmerksam zu machen, die Gonseth nach seinem Zürcher Aufenthalt auf nationaler und internationaler Ebene eingenommen hat.

Auf der nationalen Ebene hat er einen zweifachen Einfluß ausgeübt, und zwar einerseits durch den Eindruck, welchen seine starke Persönlichkeit in Zürich, wie übrigens auch schon in Bern, bei unzähligen Schülern hinterlassen hat, die mehrheitlich Ingenieure, zu einem großen Teil aber auch Lehrer in der ganzen Schweiz geworden sind. Andererseits hat seine zweite Einflußnahme sich teilweise gegen ihn selbst gewendet. Sie bestand darin, daß Gonseth Lehrbücher für den Mathematikunterricht verfaßte; S. Gagnebin und mein Vater haben zu dieser Aufgabe auch beigetragen. In diesem Unterfangen stieß Gonseth mit seinen festumrissenen Vorstellungen, und obgleich ihm mehrere Mitarbeiter zur Seite standen, auf heftigen Widerstand. Er selbst besaß kaum Erfahrungen im Sekundarschulunterricht und hat wahrscheinlich die Psychologie des Heranwachsenden in der Praxis nicht beherrscht. Er war eher befähigt, bereits erwachsene junge Leute zu leiten. Da konnte sich seine Begabung zum großzügigen Lehrer voll entfalten. Seine mathematisch-pädagogischen Arbeiten wiederum hinterließen nicht die erwarteten Spuren. Es war vielmehr auf internationaler Ebene, daß Gonseth von den Dreißigerjahren an eine hervorragende Rolle zu spielen begann. Wohlverstanden, sein Name war schon über die Landesgrenzen hinaus vorgedrungen; er hatte 1926 in Paris die Grundlagen der Mathematik veröffentlicht, welche vom historischen Werdegang des Dilemmas des Brouwerschen Intuitionismus handelten, und er hatte auch mehrere technische Arbeiten verfaßt, darunter, gemeinsam mit G. Juvet, zwei Aufsätze über die Möglichkeit, die Schrödinger-Gleichung im fünfdimensionalen relativistischen Formalismus auszudrücken. Seine internationale Tätigkeit beginnt aber eigentlich mit seiner Niederlassung in Zürich. Es sind drei Tätigkeitskreise zu unterscheiden.

Der erste besteht in der Teilnahme an internationalen Begegnungen, insbesondere im Gebiet der Philosophie der Wissenschaften, sei es, daß er sich zu Kongressen begab, oder daß er selber solche, vor allem in Zürich, organisierte oder anregte. So hat er zum Beispiel am berühmten internationalen Kongre $B$ für wissenschaftliche Philosophie 1935 in Paris (vgl. Kongreßakten 1936) - dem ersten dieses Namens, mit welchem auf die Initiative des Wienerkreises hin die "Einheit der Wissenschaft" erstmals demonstriert werden sollte - die Idee einer Logik als „Physik des beliebigen Objektes" lanciert (wiederaufgenommen in Was ist Logik?, Paris 1937). Bei diesem Anlaß nahm Gonseth einen Platz unter den Koryphäen ein, d.h. Gelehrten wie Niels Bohr, P. W. Bridgman, R. Carnap, Jakob Clay, F. Enriques, Ph. Franck, Jørgen Jørgensen, J. Lukasiewicz, R. von Mises, Hans Reichenbach, Bertrand Russell, Moritz Schlick und viele andere, zu denen sich später Barzin, Beth, Bouligand, Destouches und schließlich Perelman, Popper u.a.m. gesellten. Von nun an sollte Gonseth auf der internationalen Bühne der Philosophie der Wissen- 
schaften eine originelle Tendenz vertreten, welche sich weder der formellen Logik anschloB, noch dem Neopositivismus, noch dem Operationalismus, noch dem (logischen oder sonstigen) Empirismus, noch dem Rationalismus, noch dem (dialektischen oder sonstigen) Materialismus, noch der (übrigens erst später entstandenen) analytischen Philosophie, und auch nicht dem Wittgenstein, den Gonseth nebenbei bemerkt nicht gelesen hatte, denn ich selbst habe ihn ihm erklärt (zu einer Zeit, als erst vom Tractatus die Rede war). Gonseth's ,höchsteigene" Philosophie bestand in der Überlegung, daß, auf welchem Wege wir auch immer an die Wissenschaft herantreten, wir dort eine bereits formulierte Kenntnis antreffen, welche uns eine Idee dessen, was ist, vermittelt, und daß wir demzufolge einfach nur von dieser vorgängigen Idee ausgehen können, um weiterzukommen, und daß jeder beliebige Punkt, welchen wir erreicht haben, wiederum stets nur eine Vorstufe darstellt zu dem, was wir später Besseres finden werden: kurz, da $B$ das, was wir in einem gegenwärtigen Zeitpunkt denken, dementsprechend revidierbar ist. Dies ist die offene Philosophie klassiert in den Rang einer fundamentalen Methodologie.

Wir werden nicht alle Begegnungen hier erwähnen. Da gab es den Descartes Kongreß, dessen Besuch für , die Welt“ praktisch ein Muß war. Da gab es die ersten Kolloquien des Internationalen Instituts für Philosophie, welche sich allerdings mit Problemen befaßten, die Gonseth zu jener Zeit nicht beschäftigten, seinen Geist aber vielleicht auf andere Fragenkomplexe hinlenkten ...

Im Jahre 1938 organisierte Gonseth die ersten „Züricher Gespräche“, welche er mit dem Thema ,Grundlagen und Methode der mathematischen Wissenschaften " überschrieb. Die Methode war also von vornherein dabei, und in seinen Schlußfolgerungen gebraucht er das Wort Pertinenz, das den deutschen Begriff der Sachlichkeit wiedergeben soll (und nicht der Objektivität, die etwas anderes ist), - ein Wort, das ich viel später in einem universellen Sinne wieder aufgenommen habe, - und er schafft die Idee der Dialektik als Prinzip der Vereinheitlichung, denn, sagt er, es gibt unter Experten abweichende Ansichten über ein und dieselbe Materie, und weil man die Auffassung eines Experten nicht zugunsten derjenigen dieses oder jenes andern verwerfen kann, haben sie folglich alle recht, jeder aus seiner Perspektive. Anstatt ihnen irgendeine Einseitigkeit vorzuwerfen, muß man ihre Perspektiven durch eine Dialektik der Bedeutung der zur Diskussion stehenden Dinge, der Ziele, welche man verfolgt, und deren sprachlicher Bewältigungsmöglichkeiten vereinheitlichen. Das tut jedoch die Logik nicht, denn sie entbehrt dieser drei Elemente; sie ist symbolisch und nur symbolisch. Die Dialektik ist folglich etwas anderes als die Logik. Kurz nach den genannten ,,Gesprächen" brach der Zweite Weltkrieg aus. Gonseth hatte diesen Krieg deutlich kommen fühlen. Er gehörte zu jenen, die sich moralisch auf das Unheil vorbereitet hatten, welches über so viele Menschen und darunter so zahlreiche Gelehrte hereinbrechen würde. 
Nach dem Krieg war er eine der Stützen kommender Zusammenkünfte. Er begab sich überall hin, außer nach Amerika. Er sprach kein Englisch und lehnte seinerzeit sogar ab, daß ich ihn in die Vereinigten Staaten begleite, um ihm (physisch wegen seiner Sehbehinderung und als Dolmetscher) behilflich zu sein; er hätte dort Gelegenheit gehabt, die Achtung, welche ihm sonst überall zuteil wurde, noch vertieft zu erleben.

Obwohl ich Gonseth früher schon durch meinen Vater kennengelernt hatte, trat ich doch erst nach 1939 häufig mit ihm in Verbindung, und diese Kontakte blieben bis zuletzt sehr lebendig, mit Ausnahme einer Unterbrechung, welche auf ein Mißverständnis zurückging, von dem ich noch berichten werde. $\mathrm{Da}$ ich der Westschweizer Tradition, welche ich weiter oben beschrieben habe, verbunden war, dort auch in die philosophische Betrachtungsweise der Wissenschaften eingeführt, aber beruflich als Physiker und Kosmologe mehr denn als reiner Mathematiker ausgebildet, aufbauend auf einer doppelten, französisch-angelsächsischen Grundlage, und schließlich in der Schule von Niels Bohr geformt worden war, sah ich die Dinge unter einem ganz anderen Blickwinkel als Gonseth. Für mich ist die Mathematik ein Produkt des homo ludens und nicht des homo faber, weshalb Gonseth und ich trotz wirkungsvoller und sehr fruchtbarer Diskussionen uns nie ganz einig waren. Gonseth war wesentlich älter als ich; er hielt mit dem ganzen Gewicht seiner Persönlichkeit an seinen Meinungen fest und glaubte sich in der Physik beschlagen, obschon er nie wirklich beruflich in diesem Gebiet geforscht hatte. (Juvet, selbst vom Schlage jener Gelehrten in der Art eines Hilbert und eines Poincaré, welche in der Analyse der mathematischen Methoden der Physik ihre Sporen abverdient hatten, hatte Gonseth in die Physik eingeführt.) Gonseth fühlte den radikalen Unterschied nicht, der zwischen dem Mathematischen und dem Physikalischen besteht. Er sah die Mathematik als eine gereinigte Physik, weil, sagte er, die Mathematik aus der Erfahrung abstrahiert wird.

In den ersten Jahren unserer näheren Bekanntschaft - vor allem während des Krieges 1939 bis 1945 - fanden Gespräche statt (oft zu zweit, doch manchmal waren wir mehrere, meistens zu Gast an der Goldauerstraße 60 in Zürich, wo Frau Gonseth gelbe Zitronentörtchen anzubieten pflegte), Gespräche, aus denen hervorging, daß die Interessen Gonseth's eine neue Wendung vollzogen: Mathematik und Naturwissenschaften befriedigten seinen Wissensdrang nicht mehr zur Genüge. Ich entsinne mich einer Diskussion über die außergewöhnlichen Begabungen der Negersängerin Marion Anderson, welche dem musikliebenden Publikum Europas durch Ernest Ansermet vorgestellt worden war, und über die axiologischen Grundlagen ihrer Kunst. Da Gonseth nicht sehen konnte, war er kaum imstande die Künste zu schätzen, deren Genuß das Sehen voraussetzt, und so enthielt er sich einer Stellungnahme; ausgerichtet auf Musik und Poesie wie er war, mußte er einsehen, daß es hier um eine wesentliche Komponente der menschlichen Geistestätigkeit ging, obwohl 
er über dieses Thema nur einen einzigen Aufsatz (übrigens gemeinsam mit seinem Sohn) verfaßt hat.

Allerdings hat er sich angesichts einer sehr ehrwürdigen, jedoch seinem Denken entgegenlaufenden Denktradition zugleich angezogen und zur Opposition angetrieben gefühlt: der Tradition des katholischen und insbesondere des neo-thomistischen Denkens. Die Kontroversen, welche er zum Beispiel an den Kongressen der französischsprachigen Philosophen unterhielt, wo zahlreiche Kleriker zu treffen waren (so Pater Ysaye, S. J., mit seinen scharfen Repliken), genügten ihm nicht. Er wollte eine echte kontradiktorische Auseinandersetzung. Zweimal gelang es ihm, eine solche zu realisieren: das erste Mal 1951 in Rom über neuscholastische Philosophie und offene Philosophie (Gesprächsleiter Mgr. Raffa), und das zweite Mal 1957 bei den Gesprächen über die Metaphysik und die Öffnung gegen die Erfahrung (man beachte die zweimalige Verwendung des Begriffes „offen“, bzw. ,Öffnung“). Hauptsächlich von daher stammt seine Gewohnheit, gewisse Philosophen als „Metaphysiker" zu bezeichnen, deren Denken er achtete, ja sogar schätzte, die sich aber seiner Meinung nicht anschließen wollten, weil sie der Wirklichkeit einen wesentlichen ontologischen Status gaben, den er, Gonseth, nicht anerkennen wollte. So hat er auch mich seit jener Zeit als Metaphysiker behandelt. Ich fühle mich versucht $z u$ sagen, da $B$ es Gonseth nie gelungen ist, sich ein klares Bild davon zu machen, was ein metaphysisches Erlebnis bedeutet, ungeachtet der Tatsache, daß er sich durch die Metaphysik angezogen fühlte. Er akzeptierte, daß Wissenschaft und Philosophie ein Ganzes bilden (wie der Kardinal Mercier ausgedrückt hatte), doch suchte er erfolglos nach den Evidenzen der Metaphysilk. Seine Zurückhaltung gegenüber der Metaphysik war verschieden von der grundsätzlichen Verurteilung der Metaphysik wie sie bei Jean Piaget, oder ganz allgemein bei den Positivisten aller Färbungen $\mathrm{zu}$ finden ist.

Die Konfrontationen in Rom oder auch anderswo haben Gonseth das Beiwort Polemiker eingetragen: gegen bestimmte Philosophien rationalistischer, positivistischer u.a. Prägung. Ein Polemiker war er ohne Zweifel. Er ging manchmal sogar recht weit in seinen Polemiken, und da er wegen seiner Augen nicht viel lesen konnte, war seine Bildung nicht unbegrenzt, und er lief ab und $\mathrm{zu}$ Gefahr, sich zu vergaloppieren. Allerdings war er zu intelligent, um sich in solchen Situationen nicht aus der Affäre ziehen zu können, und Samuel Gagnebin, in der Philosophie sehr bewandert, hat ihm als ,Korrektor“ große Dienste geleistet.

Aber lassen wir das Thema der Teilnahme an Zusammenkünften. Fügen wir nur noch bei, da $B$ er sich nicht damit $z \mathfrak{u}$ begnügen liebte zuzuhören; er wollte intervenieren, als wäre er von einem Bedürfnis getrieben, sich selbst eine überlegene Position gegenüber den anderen zu bestätigen, - eine Art Aggressivität, die er immerhin selbst zu mäßigen verstand.

Die zweite erinnerungswerte Manifestation seiner Persönlichkeit ist die ihm zu verdankende Förderung von internationalen Organisationen oder Institutionen. Er sah die Einheit „Wissenschaft und Philosophie“ un- 
weigerlich verbunden mit den Wissenschaften einerseits und der Philosophie andererseits, und er hat alles daran gesetzt, da $B$ diese doppelte Bindung sich auch administrativ auswirkte. Man hat vergessen, da $B$ man Gonseth die Existenz der Abteilung für Logik und Philosophie der Wissenschaften verdankt, welche eines der beiden Tätigkeitsgebiete der Internationalen Union für Geschichte und Philosophie der Wissenschaften trägt (IUHPS; während zwei weitere Schweizer eine wichtige Rolle in der Abteilung für Geschichte der Wissenschaften gespielt haben: Arnold Reymond und William Schopfer). Diese Union ist dem ICSU, dem Internationalen Rat der wissenschaftlichen Unionen, angegliedert. Parallel dazu hatte Gonseth eine Internationale Gesellschaft für Logik und Philosophie der Wissenschaften gegründet, welche er der Internationalen Föderation philosophischer Gesellschaften (FISP) anzugliedern bestrebt war, die ihrerseits dem CIPSH, dem Internationalen Rat für Philosophie und Geisteswissenschaften angeschlossen ist. Dadurch waren Naturwissenschaft und Geisteswissenschaft verbunden, und er sah darin eine durchaus konkrete Bindung. Doch die Geschichte kümmert sich nicht um die Wünsche der Menschen; die genannte Abteilung wurde alsbald fast ausschließlich eine Organisation für formelle Logik und angelsächsische analytische Philosophie, und Gonseth war sehr enttäuscht darüber: Wo sind die Wissenschaftsphilosophen? rief er in den Sechzigerjahren in meiner Gegenwart einmal aus. Der alte Wienerkreis konnte einer wirklichen Wissenschaftsphilosophie keine verdauliche Nahrung zuführen; der Wienerkreis war destruktiv, und seine Versuche, konstruktiv zu sein, haben alle versagt, während die wirklichen Wissenschaftsphilosophen, d.h. jene, die die Wissenschaft (oder doch wenigstens eine Einzelwissenschaft) durch die Ausübung eines entsprechenden Berufes kennen, sehr selten geworden sind (es gab einen oder zwei im Wienerkreis, namentlich $\mathrm{Ph}$. Franck und H. Reichenbach, und von jenen, die sich vor dem Krieg, von der Physik ausgehend, völlig unabhängig von jeder vorgängigen philosophischen Doktrin ausgebildet hatten, bleiben eigentlich nur Jean Piaget, Henry Margenau, Jean-Louis Destouches, Wolfgang Yourgrau, Martin StrauB, Carl Friedrich von Weizsäcker, Mario Bunge und ich selbst). Das hat Gonseth alsbald bemerkt: eine erste Enttäuschung. Die zweite Enttäuschung war, daß die internationale Gesellschaft, welche er gegründet hatte, gefährdet war; die sogenannten nationalen Sektionen hatten bald keine Mitglieder mehr, und eines Tages bat mich Gonseth darum, einen Versuch zu unternehmen, diese Gesellschaft aus der Asche wieder erstehen zu lassen, um ihr - wie er es nannte - einen großen Aufschwung in Richtung einer echten Wissenschaftsphilosophie zu geben. Aber der Vogel Phönix ist ein Mythos, entgegnete ich, und die Wirklichkeit richtet sich nicht nach unseren Wünschen. Es war besser, den Tatsachen ins Auge zu sehen. Die IUHPS blühte und blüht heute noch; ihre Abteilung für Logik und Philosophie der Wissenschaften hat in der Folge weltweite Kongresse organisiert (Gonseth hatte den seinigen in Zürich durchgeführt), ohne sich weiter um die Bedingungen zu kümmern, 
welche Gonseth hätte erfüllt sehen wollen. Die Sache war leider dahin, und Gonseth hat sich damit abgefunden. Inzwischen hat er viel zum Aufschwung beigetragen, den die internationale Akademie für Wissenschaftsphilosophie genommen hat, eine der Sektionen des Instituts für theoretische Wissenschaften (die andere Sektion befaßt sich mit den Religionswissenschaften), die Pater Dockx ins Leben gerufen hat. Im übrigen hat sich Gonseth mehr und mehr den Betrachtungen darüber gewidmet, wie seine Auffassung von Dialektik auf ein immer weiteres Verständnis der Probleme der Menschheit anzuwenden sei. Mathematik und Wirklichkeit waren nur noch Ausgangspunkte seiner Laufbahn; der Höhepunkt seines Gedankenbaus stand vielfältigen Problemen offen.

Dies veranlaßt mich, vom dritten konkreten Tätigkeitskreis Gonseth's zu sprechen: die Gründung der Zeitschrift DIALECTICA. Sie geht auf 1947 zurück und beginnt (Geleitwort zu No 1) mit den folgenden Worten: „So manche sagen heute: Wir lehnen den Geist dieses Jahrhunderts ab ..."; Widerrede: ,Wir entscheiden uns für die Beteiligung und ihre Gefahren." Erinnern wir in diesem Zusammenhang daran, daß die Idee der Beteiligung, des Engagiertseins, nicht, wie man oft annimmt, von Jean Paul Sartre eingeführt wurde, sondern von Denis de Rougemont, den wir gewissermaßen als literarisches Gegenstück zum wissenschaftlich ausgerichteten Gonseth betrachten können.

Obwohl Gonseth darauf bestand, zwei Kollegen für die eigentliche Redaktion und eine Anzahl weiterer in einem Redaktionskomitee beizuziehen, was seiner Zeitschrift den schon zu Beginn angestrebten internationalen Charakter verleihen sollte, hat er die Leitung der DIALECTICA in Wahrheit stets in der Hand behalten und auch die meisten Nummern persönlich inspiriert.

DIALECTICA wurde 19 Jahre lang vom Verlangshaus Le Griffon herausgegeben, einer Gründung Marcel Joray's, auch er Jurassier, ursprünglich Botaniker und Pädagoge aus der Schule des großen Physiologen und Naturwissenschaftshistorikers William Schopfer, der seinerzeit der Genfer Tradition entstammte und in Bern den Lehrstuhl für Botanik innehatte. Ohne Joray wäre selbstverständlich DIALECTICA dennoch erschienen, denn Gonseth war nicht aufzuhalten, wohl aber hätte diese Herausgabe vielleicht nicht jenen Grad von ästhetischer Vollkommenheit erreicht, den man ihr von Anfang an zugestehen mußte. Le Griffon hat zu Beginn wissenschaftliche Werke publiziert, denen Gonseth übrigens kein Unbekannter war. Damals, kurz nach dem Krieg, als Frankreich große Mühe hatte, wieder ein Lager an derartigen Büchern aufzubauen, vermochte die Schweiz eine große Lücke zu füllen. Dort, wo Juvet als Bahnbrecher vor dem Krieg versagt hatte, hat Gonseth später Erfolg gehabt. Seither hat Le Griffon die Wissenschaft aufgegeben, um sich nur noch der Herausgabe von Kunstbänden zu widmen, einer Leidenschaft ihres Direktors. Auch DIALECTICA ist auf andere Buchpressen übergegangen.

Wenn man versucht festzustellen, was DIALECTICA Besonderes publiziert hat, stöBt man auf Schwierigkeiten. Man findet in der Tat ein 
bißchen von allem. Es geht wirklich um ein allseitiges Engagement, mit dem Hintergedanken, daß sich die Dialektik, welche Gonseth im Auge hatte, ohnehin aus sich selbst heraus manifestieren würde, und notfalls auch dank einem Hinweis des Herausgebers oder aufgrund von Schlußfolgerungen $\mathrm{zu}$ den Streitgesprächen der verschiedensten Autoren. Oftmals behandelt ein Heft, oder sogar mehrere in einem Band vereinte Hefte, ein zentrales Thema oder reproduziert die Debatten anläßlich einer philosophischen Zusammenkunft. Als Beispiel möchte ich die Doppelnummer zitieren, welche Gonseth zu seinem 70. Geburtstag gewidmet wurde, sowie die Kongreßakten der Gespräche des Internationalen Instituts für Philosophie in Oberhofen. Die Dialektik ist in dieser Zeitschrift eigentlich vor allem durch das Mittel des Dialogs und des seinem Initiator so teuren - kontradiktorischen Wortstreits hervorgetreten. Um dies zu illustrieren, war mir daran gelegen, dem Jubiläumsband von 1959 (erschienen 1960), neben einem ersten, Gonseth selbst gewidmeten Beitrag, einen zweiten, Frau Gonseth gewidmeten Beitrag erscheinen zu lassen, der absichtlich in der Form eines platonischen Dialogs verfaßt war, in welchem die Rolle des Gonseth so lieben ,,idoneum" einer "Dame" zukommt, welche am Ende des Dialogs schließlich mit ihrem Gesprächspartner, dem Philosophen, die Rolle tauscht: genau, was Gonseth mit seinen Gesprächspartnern zu tun wünschte. Er hat seine eigene Philosophie eine idoneistische Philosophie genannt. Zu jener Zeit haben Gonseth und ich uns einander sehr nahe gefühlt. Wir haben uns sehr oft getroffen, und damals hat er gewünscht, daß ich nach und nach die Tradition der internationalen Beziehungen, die er so meisterhaft eingeführt hatte, auf meinen Schultern weitertrage.

Dann folgte eine weniger glückliche Zeit, nach dem Erscheinen seines Buches über Das Problem der Zeit. Ein Beitrag zur Methodologie der Forschung (Neuenburg 1964). In einem Artikel, den die STUDIA PHILOSOPHICA publizierten, sah ich mich veranlaßt zu bemerken, daß dieses Buch Behauptungen enthalte, welche die Physik nicht annehmen könne. Abgesehen davon habe ich das Buch sehr gelobt, insbesondere die sehr originelle Idee, einen ganzen Teil der ,dialektischen“ Analyse der Zeit auf einer Untersuchung des Verbums und des Adverbs beruhen zu lassen. Diese Idee einer grammatikalischen Analyse der Zeitlichkeit ist ausgesprochen bezeichnend für Gonseth. Die Streitigkeiten unter Gelehrten sind, vor allem wenn es sich um Philosophen handelt, ein Bestandteil ihrer Laufbahn. Sie stärken ihren Charakter und, wenn sie in einer gegenseitigen Anerkennung enden, sind sie im großen und ganzen heilsam. Die Rede, welche ich als ehemaliger Rektor namens der Universität Bern an Gonseth zu dessen 80. Geburtstag gerichtet habe, hat dies, glaube ich, zur Genüge bewiesen.

Während ungefähr 10 Jahren wohnte Gonseth in Lausanne, wo er nebst persönlichen Gründen - gehofft hatte, eine seinem Denken geneigte Atmosphäre und einen Kreis von Diskussionspartnern zu finden. Denn in Zürich fühlte er sich zunehmend einsamer. Doch auch da wieder muß er 
vermutlich eine gewisse Enttäuschung verspürt haben. Die Umstände in der Westschweiz waren keineswegs mehr jene der weiter oben beschriebenen heroischen Epoche. Gegen 1965 wurde Wissenschaftstheorie nicht mehr so getrieben wie seinerzeit um 1925 die Wissenschaftsphilosophie. Vierzig Jahre, mit einem Weltkrieg und mit dem Hegemonieanspruch des wissenschaftsphilosophischen angelsächsischen Denkens, haben die Welt nicht nur auf politischer, sondern ebensosehr auf intellektueller Ebene gewandelt, und dazu kam bereits die Strömung der marxistischen Philosophie. Da war Lausanne nicht besser als Zürich. Der marxistische Schub und die Studentenrevolte waren geeignete Phänomene, um Gonseth's Interesse zu wecken, denn hier ging es nicht um lokale Erscheinungen, sondern um solche von menschlichem Gewicht.

Gonseth hat immer Gesprächspartner benötigt, die er zum Sprechen brachte, um über die Konvergenzen und die Divergenzen hinaus das Wirken der offenen Philosophie festzustellen. Wie er in seiner Einführung zu Determinismus und freier Wille (eine der ersten Publikationen von Le Griffon: Gespräche zwischen jungen Gelehrten, die er in Zürich präsentiert hatte, erschienen 1944) gesagt hat, ,geht der Widerspruch der Wahrheit voraus und verkündet sie", und Samuel Gagnebin, Gast bei diesen Gesprächen, schloß: ,Gleichermaßen dem wirksamen Wert der vernünftigen Ordnungen und deren Relativität bezüglich unseres Geistes Rechnung tragen heißt genau jene Dialektik gründen, welche Gonseth seit langem mit dem Namen Idoneismus bezeichnet hat."

? Die Ubersetzung der französischen Originalfassung ins Deutsche wurde von Maja Svilar besorgt.

Adresse des Autors:

Prof. Dr. André Mercier, Institut für exakte Wissenschaften der Universität Sidlerstraße 5, CH-3012 Bern, Schweiz 